\title{
Perfectness of Kirillov-Reshetikhin crystals for nonexceptional types
}

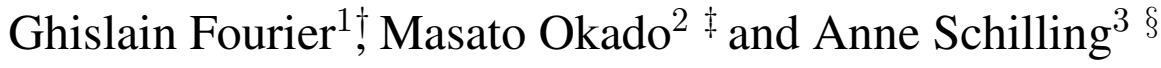 \\ ${ }^{1}$ Mathematisches Institut der Universität zu Köln, Weyertal 86-90, 50931 Köln, Germany \\ ${ }^{2}$ Department of Mathematical Science, Graduate School of Engineering Science, Osaka University, Toyonaka, Osaka \\ 560-8531, Japan \\ ${ }^{3}$ Department of Mathematics, University of California, One Shields Avenue, Davis, CA 95616-8633, U.S.A.
}

\begin{abstract}
For nonexceptional types, we prove a conjecture of Hatayama et al. about the prefectness of KirillovReshetikhin crystals.

Résumé. Pour les types non-exceptionnels, on démontre une conjecture de Hatayama et al. concernant la perfection des cristaux de Kirillov-Reshetikhin.
\end{abstract}

Keywords: Crystal bases, combinatorial models for Kirillov-Reshetikhin crystals, perfectness

\section{Introduction}

Kirillov-Reshetikhin (KR) crystals $B^{r, s}$ are crystals corresponding to finite-dimensional $U_{q}^{\prime}(\mathfrak{g})$-modules [3, [4], where $\mathfrak{g}$ is an affine Kac-Moody algebra. Recently, a lot of progress has been made regarding long outstanding problems concerning these crystals which appear in mathematical physics and the path realization of affine highest weight crystals [13]. In [20, 21] the existence of KR crystals was shown. In [5] a major step in understanding these crystals was provided by giving explicit combinatorial realizations for all nonexceptional types. This abstract is based on [5, 6]. We prove a conjecture of Hatayama, Kuniba, Okado, Takagu, and Tsuboi [8, Conjecture 2.1] about the perfectness of these KR crystals.

Conjecture 1.1 [8. Conjecture 2.1] The Kirillov-Reshetikhin crystal $B^{r, s}$ is perfect if and only if $\frac{s}{c_{r}}$ is an integer with $c_{r}$ as in Table 1 If $B^{r, s}$ is perfect, its level is $\frac{s}{c_{r}}$.

In [14], this conjecture was proven for all $B^{r, s}$ for type $A_{n}^{(1)}$, for $B^{1, s}$ for nonexceptional types (except for type $C_{n}^{(1)}$ ), for $B^{n-1, s}, B^{n, s}$ of type $D_{n}^{(1)}$, and $B^{n, s}$ for types $C_{n}^{(1)}$ and $D_{n+1}^{(2)}$. When the highest weight is given by the highest root, level-1 perfect crystals were constructed in [1]. For $1 \leq r \leq n-2$

\footnotetext{
${ }^{\dagger}$ Supported in part by DARPA and AFOSR through the grant FA9550-07-1-0543 and by the DFG-Projekt "Kombinatorische Beschreibung von Macdonald und Kostka-Foulkes Polynomen".

¥Supported by grant JSPS 20540016 .

$\S$ Supported in part by the NSF grants DMS-0501101, DMS-0652641, and DMS-0652652. 


\begin{tabular}{|c|c|}
\hline & $\left(c_{1}, \ldots, c_{n}\right)$ \\
\hline \hline$A_{n}^{(1)}, D_{n}^{(1)}, A_{2 n-1}^{(2)}, A_{2 n}^{(2)}, D_{n+1}^{(2)}$ & $(1, \ldots, 1)$ \\
\hline$B_{n}^{(1)}$ & $(1, \ldots, 1,2)$ \\
\hline$C_{n}^{(1)}$ & $(2, \ldots, 2,1)$ \\
\hline
\end{tabular}

Tab. 1: List of $c_{r}$

for type $D_{n}^{(1)}, 1 \leq r \leq n-1$ for type $B_{n}^{(1)}$, and $1 \leq r \leq n$ for type $A_{2 n-1}^{(2)}$, the conjecture was proved in [22]. The case $G_{2}^{(1)}$ and $r=1$ was treated in [24] and the case $D_{4}^{(3)}$ and $r=1$ was treated in [16]. Naito and Sagaki [18] showed that the conjecture holds for twisted algebras, if it is true for the untwisted simply-laced cases.

In this paper we prove Conjecture 1.1 in general for nonexceptional types.

Theorem 1.2 If $\mathfrak{g}$ is of nonexceptional type, Conjecture 1.1 is true.

The paper is organized as follows. In Section 2 we give basic notation and the definition of perfectness in Definition 2.1. In Section 3 we review the realizations of the KR crystals of nonexceptional types as recently provided in [5]. Section 4 is reserved for the proof of Theorem 1.2 and an explicit description of the minimal elements $B_{\mathrm{min}}^{r, c_{r} s}$ of the perfect crystals. A long version of this article containing further details and examples is available at [6].

\section{Definitions and perfectness}

We follow the notation of [12, 5]. Let $\mathcal{B}$ be a $U_{q}^{\prime}(\mathfrak{g})$-crystal [15]. Denote by $\alpha_{i}$ and $\Lambda_{i}$ for $i \in I$ the simple roots and fundamental weights and by $c$ the canoncial central element associated to $\mathfrak{g}$, where $I$ is the index set of the Dynkin diagram of $\mathfrak{g}$ (see Table 2). Let $P=\oplus_{i \in I} \mathbb{Z} \Lambda_{i}$ be the weight lattice of $\mathfrak{g}$ and $P^{+}$the set of dominant weights. For a positive integer $\ell$, the set of level $\ell$ weights is

$$
P_{\ell}^{+}=\left\{\Lambda \in P^{+} \mid \operatorname{lev}(\Lambda)=\ell\right\} .
$$

where $\operatorname{lev}(\Lambda):=\Lambda(c)$. The set of level-0 weights is denoted by $P_{0}$. We identify dominant weights with partitions; each $\Lambda_{i}$ yields a column of height $i$ (except for spin nodes). For more details, please consult [11].

We denote by $f_{i}, e_{i}: \mathcal{B} \rightarrow \mathcal{B} \cup\{\emptyset\}$ for $i \in I$ the Kashiwara operators and by wt $: \mathcal{B} \rightarrow P$ the weight function on the crystal. For $b \in \mathcal{B}$ we define $\varepsilon_{i}(b)=\max \left\{k \mid e_{i}^{k}(b) \neq \emptyset\right\}, \varphi_{i}(b)=\max \left\{k \mid f_{i}^{k}(b) \neq \emptyset\right\}$, and

$$
\varepsilon(b)=\sum_{i \in I} \varepsilon_{i}(b) \Lambda_{i} \quad \text { and } \quad \varphi(b)=\sum_{i \in I} \varphi_{i}(b) \Lambda_{i} .
$$

Next we define perfect crystals, see for example [11].

Definition 2.1 For a positive integer $\ell>0$, a crystal $\mathcal{B}$ is called perfect crystal of level $\ell$, if the following conditions are satisfied: 


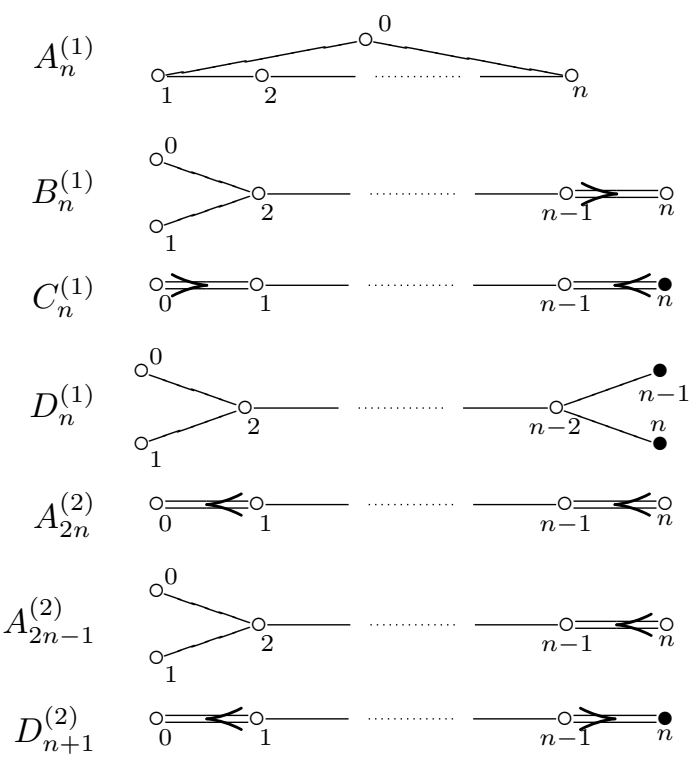

Tab. 2: Dynkin diagrams

1. $\mathcal{B}$ is isomorphic to the crystal graph of a finite-dimensional $U_{q}^{\prime}(\mathfrak{g})$-module.

2. $\mathcal{B} \otimes \mathcal{B}$ is connected.

3. There exists $a \lambda \in P_{0}$, such that wt $(\mathcal{B}) \subset \lambda+\sum_{i \in I \backslash\{0\}} \mathbb{Z}_{\leq 0} \alpha_{i}$ and there is a unique element in $\mathcal{B}$ of classical weight $\lambda$.

4. $\forall b \in \mathcal{B}, \operatorname{lev}(\varepsilon(b)) \geq \ell$.

5. $\forall \Lambda \in P_{\ell}^{+}$, there exist unique elements $b_{\Lambda}, b^{\Lambda} \in \mathcal{B}$, such that

$$
\varepsilon\left(b_{\Lambda}\right)=\Lambda=\varphi\left(b^{\Lambda}\right) .
$$

We denote by $\mathcal{B}_{\min }$ the set of minimal elements in $\mathcal{B}$, namely

$$
\mathcal{B}_{\text {min }}=\{b \in \mathcal{B} \mid \operatorname{lev}(\varepsilon(b))=\ell\} .
$$

Note that condition (5) of Definition 2.1 ensures that $\varepsilon, \varphi: \mathcal{B}_{\min } \rightarrow P_{\ell}^{+}$are bijections. They induce an automorphism $\tau=\varepsilon \circ \varphi^{-1}$ on $P_{\ell}^{+}$.

In [22, 5] \pm -diagrams were introduced, which describe the branching $X_{n} \rightarrow X_{n-1}$ where $X_{n}=$ $B_{n}, C_{n}, D_{n}$. A \pm -diagram $P$ of shape $\Lambda / \lambda$ is a sequence of partitions $\lambda \subset \mu \subset \Lambda$ such that $\Lambda / \mu$ and $\mu / \lambda$ are horizontal strips (i.e. every column contains at most one box). We depict this \pm -diagram by the skew 
tableau of shape $\Lambda / \lambda$ in which the cells of $\mu / \lambda$ are filled with the symbol + and those of $\Lambda / \mu$ are filled with the symbol - . There are further type specific rules which can be found in [5, Section 3.2]. There exists a bijection $\Phi$ between \pm -diagrams and the $X_{n-1}$-highest weight vectors inside the $X_{n}$ crystal of highest weight $\Lambda$.

\section{Realization of KR-crystals}

Throughout the paper we use the realization of $B^{r, s}$ as given in [5, 21, 22]. In this section we briefly recall the main constructions.

\subsection{KR crystals of type $A_{n}^{(1)}$}

Let $\Lambda=\ell_{0} \Lambda_{0}+\ell_{1} \Lambda_{1}+\cdots+\ell_{n} \Lambda_{n}$ be a dominant weight. Then the level is given by

$$
\operatorname{lev}(\Lambda)=\ell_{0}+\cdots+\ell_{n}
$$

A combinatorial description of $B^{r, s}$ of type $A_{n}^{(1)}$ was provided by Shimozono [23]. As a $\{1,2, \ldots, n\}$ crystal

$$
B^{r, s} \cong B\left(s \Lambda_{r}\right) .
$$

The Dynkin diagram of $A_{n}^{(1)}$ has a cyclic automorphism $\sigma(i)=i+1(\bmod n+1)$ which extends to the crystal in form of the promotion operator. The action of the affine crystal operators $f_{0}$ and $e_{0}$ is given by

$$
f_{0}=\sigma^{-1} \circ f_{1} \circ \sigma \quad \text { and } \quad e_{0}=\sigma^{-1} \circ e_{1} \circ \sigma .
$$

3.2 KR crystals of type $D_{n}^{(1)}, B_{n}^{(1)}, A_{2 n-1}^{(2)}$

Let $\Lambda=\ell_{0} \Lambda_{0}+\ell_{1} \Lambda_{1}+\cdots+\ell_{n} \Lambda_{n}$ be a dominant weight. Then the level is given by

$$
\begin{array}{ll}
\operatorname{lev}(\Lambda)=\ell_{0}+\ell_{1}+2 \ell_{2}+2 \ell_{3}+\cdots+2 \ell_{n-2}+\ell_{n-1}+\ell_{n} & \text { for type } D_{n}^{(1)} \\
\operatorname{lev}(\Lambda)=\ell_{0}+\ell_{1}+2 \ell_{2}+2 \ell_{3}+\cdots+2 \ell_{n-2}+2 \ell_{n-1}+\ell_{n} & \text { for type } B_{n}^{(1)} \\
\operatorname{lev}(\Lambda)=\ell_{0}+\ell_{1}+2 \ell_{2}+2 \ell_{3}+\cdots+2 \ell_{n-2}+2 \ell_{n-1}+2 \ell_{n} & \text { for type } A_{2 n-1}^{(2)}
\end{array}
$$

We have the following realization of $B^{r, s}$. Let $X_{n}=D_{n}, B_{n}, C_{n}$ be the classical subalgebra for $D_{n}^{(1)}$, $B_{n}^{(1)}, A_{2 n-1}^{(2)}$, respectively.

Definition 3.1 Let $1 \leq r \leq n-2$ for type $D_{n}^{(1)}, 1 \leq r \leq n-1$ for type $B_{n}^{(1)}$, and $1 \leq r \leq n$ for type $A_{2 n-1}^{(2)}$. Then $B^{r, s}$ is defined as follows. As an $X_{n}$-crystal

$$
B^{r, s} \cong \bigoplus_{\Lambda} B(\Lambda)
$$

where the sum runs over all dominant weights $\Lambda$ that can be obtained from $s \Lambda_{r}$ by the removal of vertical dominoes. The affine crystal operators $e_{0}$ and $f_{0}$ are defined as

$$
f_{0}=\sigma^{-1} \circ f_{1} \circ \sigma \text { and } e_{0}=\sigma^{-1} \circ e_{1} \circ \sigma,
$$

where $\sigma$ is the crystal automorphism defined in [22] Definition 4.2]. 
Definition 3.2 Let $B_{A_{2 n-1}^{(2)}}^{n, s}$ be the $A_{2 n-1}^{(2)}-K R$ crystal. Then $B^{n, s}$ of type $B_{n}^{(1)}$ is defined through the unique injective map $S: B^{n, s} \rightarrow B_{A_{2 n-1}^{(2)}}^{n, s}$ such that

$$
S\left(e_{i} b\right)=e_{i}^{m_{i}} S(b), \quad S\left(f_{i} b\right)=f_{i}^{m_{i}} S(b) \quad \text { for } i \in I
$$

where $\left(m_{i}\right)_{0 \leq i \leq n}=(2,2, \ldots, 2,1)$.

In addition, the \pm -diagrams of $A_{2 n-1}^{(2)}$ that occur in the image are precisely those which can be obtained by doubling a \pm -diagram of $B^{n, s}$ (see [5, Lemma 3.5]). $S$ induces an embedding of dominant weights of $B_{n}^{(1)}$ into dominant weights of $A_{2 n-1}^{(2)}$, namely $S\left(\Lambda_{i}\right)=m_{i} \Lambda_{i}$. It is easy to see that for any $\Lambda \in P^{+}$we have $\operatorname{lev}(S(\Lambda))=2 \operatorname{lev}(\Lambda)$ using (3.1).

For the definition of $B^{n, s}$ and $B^{n-1, s}$ of type $D_{n}^{(1)}$, see for example [5, Section 6.2].

\subsection{KR crystal of type $C_{n}^{(1)}$}

The level of a dominant $C_{n}^{(1)}$ weight $\Lambda=\ell_{0} \Lambda_{0}+\cdots+\ell_{n} \Lambda_{n}$ is given by

$$
\operatorname{lev}(\Lambda)=\ell_{0}+\cdots+\ell_{n}
$$

We use the realization of $B^{r, s}$ as the fixed point set of the automorphism $\sigma$ [22, Definition 4.2] (see Definition 3.1] inside $B_{A_{2 n+1}^{(2)}}^{r, s}$ of [5, Theorem 5.7].

Definition 3.3 For $1 \leq r<n$, the KR crystal $B^{r, s}$ of type $C_{n}^{(1)}$ is defined to be the fixed point set under $\sigma$ inside $B_{A_{2 n+1}^{(2)}}^{r, s}$ with the operators

$$
e_{i}= \begin{cases}e_{0} e_{1} & \text { for } i=0 \\ e_{i+1} & \text { for } 1 \leq i \leq n\end{cases}
$$

where the Kashiwara operators on the right act in $B_{A_{2 n+1}^{(2)}}^{r, s}$. Under the crystal embedding $S: B^{r, s} \rightarrow$ $B_{A_{2 n+1}^{(2)}}^{r, s}$ we have

$$
\Lambda_{i} \mapsto \begin{cases}\Lambda_{0}+\Lambda_{1} & \text { for } i=0, \\ \Lambda_{i+1} & \text { for } 1 \leq i \leq n .\end{cases}
$$

Under the embedding $S$, the level of $\Lambda \in P^{+}$doubles, that is $\operatorname{lev}(S(\Lambda))=2 \operatorname{lev}(\Lambda)$.

For $B^{n, s}$ of type $C_{n}^{(1)}$ we refer to [5, Section 6.1].

\subsection{KR crystals of type $A_{2 n}^{(2)}, D_{n+1}^{(2)}$}

Let $\Lambda=\ell_{0} \Lambda_{0}+\ell_{1} \Lambda_{1}+\cdots+\ell_{n} \Lambda_{n}$ be a dominant weight. The level is given by

$$
\begin{array}{ll}
\operatorname{lev}(\Lambda)=\ell_{0}+2 \ell_{1}+2 \ell_{2}+\cdots+2 \ell_{n-2}+2 \ell_{n-1}+2 \ell_{n} & \text { for type } A_{2 n}^{(2)} \\
\operatorname{lev}(\Lambda)=\ell_{0}+2 \ell_{1}+2 \ell_{2}+\cdots+2 \ell_{n-2}+2 \ell_{n-1}+\ell_{n} & \text { for type } D_{n+1}^{(2)}
\end{array}
$$


Define positive integers $m_{i}$ for $i \in I$ as follows:

$$
\left(m_{0}, m_{1}, \ldots, m_{n-1}, m_{n}\right)= \begin{cases}(1,2, \ldots, 2,2) & \text { for } A_{2 n}^{(2)} \\ (1,2, \ldots, 2,1) & \text { for } D_{n+1}^{(2)}\end{cases}
$$

Then $B^{r, s}$ can be realized as follows.

Definition 3.4 For $1 \leq r \leq n$ for $\mathfrak{g}=A_{2 n}^{(2)}, 1 \leq r<n$ for $\mathfrak{g}=D_{n+1}^{(2)}$ and $s \geq 1$, there exists a unique injective map $S: B_{\mathfrak{g}}^{r, s} \longrightarrow B_{C_{n}^{(1)}}^{r, 2 s}$ such that

$$
S\left(e_{i} b\right)=e_{i}^{m_{i}} S(b), \quad S\left(f_{i} b\right)=f_{i}^{m_{i}} S(b) \quad \text { for } i \in I .
$$

The \pm -diagrams of $C_{n}^{(1)}$ that occur in the image of $S$ are precisely those which can be obtained by doubling a \pm -diagram of $B^{r, s}$ (see [5, Lemma 3.5]). $S$ induces an embedding of dominant weights for $A_{2 n}^{(2)}, D_{n+1}^{(2)}$ into dominant weights of type $C_{n}^{(1)}$, with $S\left(\Lambda_{i}\right)=m_{i} \Lambda_{i}$. This map preserves the level of a weight, that is $\operatorname{lev}(S(\Lambda))=\operatorname{lev}(\Lambda)$.

For the case $r=n$ of type $D_{n+1}^{(2)}$ we refer to [5, Definition 6.2].

\section{Proof of Theorem 1.2}

For type $A_{n}^{(1)}$, perfectness of $B^{r, s}$ was proven in [14]. For all other types, in the case that $\frac{s}{c_{r}}$ is an integer, we need to show that the 5 defining conditions in Definition 2.1 are satisfied:

1. This was recently shown in [21].

2. This follows from [7, Corollary 6.1] under [7, Assumption 1]. Assumption 1 is satisfied except for type $A_{2 n}^{(2)}$ : The regularity of $B^{r, s}$ is ensured by (1), the existence of an automorphism $\sigma$ was proven in [5, Section 7], and the unique element $u \in B^{r, s}$ such that $\varepsilon(u)=s \Lambda_{0}$ and $\varphi(u)=s \Lambda_{\nu}$ (where $\nu=1$ for $r$ odd for types $B_{n}^{(1)}, D_{n}^{(1)}, A_{2 n-1}^{(2)}, \nu=r$ for $A_{n}^{(1)}$, and $\nu=0$ otherwise) is given by the classically highest weight element in the component $B(0)$ for $\nu=0, B\left(s \Lambda_{1}\right)$ for $\nu=1$, and $B\left(s \Lambda_{r}\right)$ for $\nu=r$. Note that $\Lambda_{0}=\tau\left(\Lambda_{\nu}\right)$, where $\tau=\varepsilon \circ \varphi^{-1}$. For type $A_{2 n}^{(2)}$, perfectness follows from [18].

3. The statement is true for $\lambda=s\left(\Lambda_{r}-\Lambda_{r}(c) \Lambda_{0}\right)$, which follows from the decomposition formulas [2, 9, 10, 19].

Conditions (4) and (5) will be shown in the following subsections using case by case considerations: Section 4.1 for type $A_{n}^{(1)}$, Sections 4.2 4.3 , and 4.4 for types $B_{n}^{(1)}, D_{n}^{(1)}, A_{2 n-1}^{(2)}$, Sections 4.5 and 4.6 for type $C_{n}^{(1)}$, Section 4.7 for type $A_{2 n}^{(2)}$, and Sections 4.8 and 4.9 for type $D_{n+1}^{(2)}$.

When $\frac{s}{c_{r}}$ is not an integer, we show in the subsequent sections that the minimum of the level of $\varepsilon(b)$ is the smallest integer exceeding $\frac{s}{c_{r}}$, and provide examples that contradict condition (5) of Definition 2.1 for each crystal, thereby proving that $B^{r, s}$ is not perfect. In the case that $\frac{s}{c_{r}}$ is an integer, we provide an explicit construction of the minimal elements of $B^{r, s}$. 


\subsection{Type $A_{n}^{(1)}$}

It was already proven in [14] that $B^{r, s}$ is perfect. We give below its associated automorphism $\tau$ and minimal elements. $\tau$ on $P$ is defined by

$$
\tau\left(\sum_{i=0}^{n} k_{i} \Lambda_{i}\right)=\sum_{i=0}^{n} k_{i} \Lambda_{i-r \bmod n+1} .
$$

Recall that $B^{r, s}$ is identified with the set of semistandard tableaux of $r \times s$ rectangular shape over the alphabet $\{1,2, \ldots, n+1\}$. For $b \in B^{r, s}$ let $x_{i j}=x_{i j}(b)$ denote the number of letters $j$ in the $i$-th row of $b$ for $1 \leq i \leq r, 1 \leq j \leq n+1$. Set $r^{\prime}=n+1-r$, then

$$
x_{i j}=0 \quad \text { unless } \quad i \leq j \leq i+r^{\prime} .
$$

Let $\Lambda=\sum_{i=0}^{n} \ell_{i} \Lambda_{i}$ be in $P_{s}^{+}$, that is, $\ell_{0}, \ell_{1}, \ldots, \ell_{n} \in \mathbb{Z}_{\geq 0}, \sum_{i=0}^{n} \ell_{i}=s$. Then $x_{i j}(b)$ of the minimal element $b$ such that $\varepsilon(b)=\Lambda$ is given by

$$
\begin{aligned}
x_{i i} & =\ell_{0}+\sum_{\alpha=i}^{r-1} \ell_{\alpha+r^{\prime}}, \\
x_{i j} & =\ell_{j-i} \quad\left(i<j<i+r^{\prime}\right), \\
x_{i, i+r^{\prime}} & =\sum_{\alpha=0}^{i-1} \ell_{\alpha+r^{\prime}}
\end{aligned}
$$

for $1 \leq i \leq r$.

\subsection{Types $B_{n}^{(1)}, D_{n}^{(1)}, A_{2 n-1}^{(2)}$}

Conditions (4) and (5) of Definition 2.1 for $1 \leq r \leq n-2$ for type $D_{n}^{(1)}, 1 \leq r \leq n-1$ for type $B_{n}^{(1)}$, and $1 \leq r \leq n$ for type $A_{2 n-1}^{(2)}$ were shown in [22, Section 6]. To a given fundamental weight $\Lambda_{k}$ a \pm -diagram $\operatorname{diagram}\left(\Lambda_{k}\right)$ was associated. This map can be extended to any dominant weight $\Lambda=\ell_{0} \Lambda_{0}+\cdots+\ell_{n} \Lambda_{n}$ by concatenating the columns of the \pm -diagrams of each piece. To every fundamental weight $\Lambda_{k}$ a string of operators $f\left(\Lambda_{k}\right)$ can be associated as in [22, Section 6].

The minimal element $b$ in $B^{r, s}$ that satisfies $\varepsilon(b)=\Lambda$ can now be constructed as follows

$$
b=f\left(\Lambda_{n}\right)^{\ell_{n}} \cdots f\left(\Lambda_{2}\right)^{\ell_{2}} \Phi(\operatorname{diagram}(\Lambda)) .
$$

For $\Lambda=\sum_{i=0}^{n} \ell_{i} \Lambda_{i} \in P_{s}^{+}$, we have

$$
\tau(\Lambda)= \begin{cases}\Lambda & \text { if } r \text { is even, } \\ \ell_{0} \Lambda_{1}+\ell_{1} \Lambda_{0}+\sum_{i=2}^{n} \ell_{i} \Lambda_{i} & \text { if } r \text { is odd, } \\ & \text { types } B_{n}^{(1)}, A_{2 n-1}^{(2)}, \\ \ell_{0} \Lambda_{1}+\ell_{1} \Lambda_{0}+\sum_{i=2}^{n-2} \ell_{i} \Lambda_{i}+\ell_{n-1} \Lambda_{n}+\ell_{n} \Lambda_{n-1} & \text { if } r \text { is odd, type } D_{n}^{(1)} .\end{cases}
$$




\subsection{Type $D_{n}^{(1)}$ for $r=n-1, n$}

The cases when $r=n, n-1$ for type $D_{n}^{(1)}$ were treated in [14]. We refer to [14] or [6, Section 4.3] for an explicit description of the minimal elements.

The automorphism $\tau$ is given by

$$
\tau\left(\sum_{i=0}^{n} \ell_{i} \Lambda_{i}\right)=\ell_{0} \Lambda_{n-1}+\ell_{1} \Lambda_{n}+\sum_{i=2}^{n-2} \ell_{i} \Lambda_{n-i}+ \begin{cases}\ell_{n-1} \Lambda_{0}+\ell_{n} \Lambda_{1} & n \text { even, } \\ \ell_{n-1} \Lambda_{1}+\ell_{n} \Lambda_{0} & n \text { odd. }\end{cases}
$$

\subsection{Type $B_{n}^{(1)}$ for $r=n$}

In this section we consider the perfectness of $B^{n, s}$ of type $B_{n}^{(1)}$.

Proposition 4.1 We have

$$
\begin{aligned}
& \min \left\{\operatorname{lev}(\varepsilon(b)) \mid b \in B^{n, 2 s+1}\right\} \geq s+1, \\
& \min \left\{\operatorname{lev}(\varepsilon(b)) \mid b \in B^{n, 2 s}\right\} \geq s .
\end{aligned}
$$

Proof: Suppose, there exists an element $b \in B^{n, 2 s+1}$ with $\operatorname{lev}(\varepsilon(b))=p<s+1$. Since $B^{n, 2 s+1}$ is embedded into $B_{A_{2 n-1}^{(2)}}^{n, 2 s+1}$ by Definition 3.2 this would yield an element $\tilde{b} \in B_{A_{2 n-1}^{(2)}}^{n, 2 s+1}$ with $\operatorname{lev}(\tilde{b})<2 s+1$. But this is not possible, since $B_{A_{2 n-1}^{(2)}}^{n, 2 s+1}$ is a perfect crystal of level $2 s+1$.

Suppose there exists an element $b \in B^{n, 2 s}$ with $\operatorname{lev}(\varepsilon(b))=p<s$. By the same argument one obtains a contradiction to the level of $B_{A_{2 n-1}^{(2)}}^{n, 2 s}$.

Hence to show that $B^{n, 2 s+1}$ is not perfect, it is enough to provide two elements $b_{1}, b_{2} \in B_{A_{2 n-1}^{(2)}}^{n, 2 s+1}$ which are in the realization of $B^{r, s}$ under $S$ and satisfy $\varepsilon\left(b_{1}\right)=\varepsilon\left(b_{2}\right)=\Lambda$, where $\operatorname{lev}(\Lambda)=2 s+2$. We use the notation $f_{\vec{a}}=f_{a_{1}}^{m_{1}} \cdots f_{a_{k}}^{m_{k}}$ for $\vec{a}=\left(a_{1}^{m_{1}}, \ldots, a_{k}^{m_{k}}\right)$.

Proposition 4.2 Define the following elements $b_{1}, b_{2} \in B_{A_{2 n-1}^{(2)}}^{n, 2 s+1}$ : For $n$ odd, let $P_{1}$ be the \pm -diagram corresponding to one column of height $n$ containing one + , and $2 s$ columns of height 1 each containing $a-$ sign, and $P_{2}$ the analogous \pm -diagram but with $a-i n$ the column of height $n$. Set $\vec{a}=(n,(n-$ $\left.1)^{2}, n,(n-2)^{2},(n-1)^{2}, n, \ldots, 2^{2}, \ldots,(n-1)^{2}, n\right)$ and

$$
b_{1}=f_{\vec{a}}\left(\Phi\left(P_{1}\right)\right) \quad \text { and } \quad b_{2}=f_{\vec{a}}\left(\Phi\left(P_{2}\right)\right) .
$$

For $n$ even, replace the columns of height 1 with columns of height 2 and fill them with \pm -pairs. Then $b_{1}, b_{2} \in S\left(B^{n, 2 s+1}\right)$ and $\varepsilon\left(b_{1}\right)=\varepsilon\left(b_{2}\right)=2 s \Lambda_{1}+\Lambda_{n}$, which is of level $2 s+2$.

Proof: It is clear from the construction that the \pm -diagrams corresponding to $b_{1}$ and $b_{2}$ can be obtained by doubling a $B_{n}^{(1)} \pm$-diagram (see [5, Lemma 3.5]). Hence $\Phi\left(P_{1}\right), \Phi\left(P_{2}\right) \in S\left(B^{n, 2 s+1}\right)$. The sequence $\vec{a}$ can be obtained by doubling a type $B_{n}^{(1)}$ sequence using $\left(m_{1}, m_{2}, \ldots, m_{n}\right)=(2, \ldots, 2,1)$, so by Definition $3.2 b_{1}$ and $b_{2}$ are in the image of the embedding $S$ that realizes $B^{n, 2 s+1}$. The claim that $\varepsilon\left(b_{1}\right)=\varepsilon\left(b_{2}\right)=2 s \Lambda_{1}+\Lambda_{n}$ can be checked explicitly. 
Corollary 4.3 The KR crystal $B^{n, 2 s+1}$ of type $B_{n}^{(1)}$ is not perfect.

Proof: This follows directly from Proposition 4.2 using the embedding $S$ of Definition 3.2

Proposition 4.4 There exists a bijection, induced by $\varepsilon$, from $B_{\min }^{n, 2 s}$ to $P_{s}^{+}$. Hence $B^{n, 2 s}$ is perfect of level $s$.

Proof: Let $S$ be the embedding from Definition 3.2. Then we have an induced embedding of dominant weights $\Lambda$ of $B_{n}^{(1)}$ into dominant weights of $A_{2 n-1}^{(2)}$ via the map $S$, that sends $\Lambda_{i} \mapsto m_{i} \Lambda_{i}$.

In [22, Section 6] (see Section 4.2 the minimal elements for $A_{2 n-1}^{(2)}$ were constructed by giving a \pmdiagram and a sequence from the $\{2, \ldots, n\}$-highest weight to the minimal element. Since $\left(m_{0}, \ldots, m_{n}\right)=$ $(2, \ldots, 2,1)$ and columns of height $n$ for type $A_{2 n-1}^{(2)}$ are doubled, it is clear from the construction that the \pm -diagrams corresponding to weights $S(\Lambda)$ are in the image of $S$ of \pm -diagrams for $B_{n}^{(1)}$ (see [5, Lemma 3.5]). Also, since under $S$ all weights $\Lambda_{i}$ for $1 \leq i<n$ are doubled, it follows that the sequences are "doubled" using the $m_{i}$. Hence a minimal element of $B^{n, 2 s}$ of level $s$ is in one-to-one correspondence with those minimal elements in $B_{A_{2 n-1}^{(2)}}^{n, 2 s}$ that can be obtained from doubling a \pm -diagram of $B^{n, 2 s}$. This implies that $\varepsilon$ defines a bijection between $B_{\min }^{n, 2 s}$ and $P_{s}^{+}$.

The automorphism $\tau$ of the perfect KR crystal $B^{n, 2 s}$ is given by

$$
\tau\left(\sum_{i=0}^{n} \ell_{i} \Lambda_{i}\right)= \begin{cases}\sum_{i=0}^{n} \ell_{i} \Lambda_{i} & \text { if } n \text { is even } \\ \ell_{0} \Lambda_{1}+\ell_{1} \Lambda_{0}+\sum_{i=2}^{n} \ell_{i} \Lambda_{i} & \text { if } n \text { is odd }\end{cases}
$$

\subsection{Type $C_{n}^{(1)}$}

In this section we consider $B^{r, s}$ of type $C_{n}^{(1)}$ for $r<n$.

Proposition 4.5 Let $r<n$. Then

$$
\begin{aligned}
& \min \left\{\operatorname{lev}(\varepsilon(b)) \mid b \in B^{r, 2 s+1}\right\} \geq s+1, \\
& \min \left\{\operatorname{lev}(\varepsilon(b)) \mid b \in B^{r, 2 s}\right\} \geq s
\end{aligned}
$$

Proof: By Definition 3.3 the crystal $B^{r, s}$ is realized inside $B_{A_{2 n+1}^{(2)}}^{r, s}$. The proof is similar to the proof of Proposition 4.1 for type $B_{n}^{(1)}$.

Hence to show that $B^{r, 2 s+1}$ is not perfect, it is suffices to give two elements $b_{1}, b_{2} \in B_{A_{2 n+1}^{(2)}}^{r, 2 s+1}$ that are fixed points under $\sigma$ with $\varepsilon\left(b_{1}\right)=\varepsilon\left(b_{2}\right)=\Lambda$, where $\operatorname{lev}(\Lambda)=2 s+2$.

Proposition 4.6 Let $b_{1}, b_{2} \in B_{A_{2 n+1}^{(2)}}^{r, 2 s+1}$, where $b_{1}$ consists of scolumns of the form read from bottom to top $(1,2, \ldots, r)$, s columns of the form $(\bar{r}, \overline{r-1}, \ldots, \overline{1})$, and a column $(\overline{r+1}, \ldots, \overline{2})$. In $b_{2}$ the last column is replaced by $(r+2, \ldots, 2 r+2)$ if $2 r+2 \leq n$ and $(r+2, \ldots, n, \bar{n}, \ldots, \bar{k})$ of height $n$ otherwise. Then

$$
\varepsilon\left(b_{1}\right)=\varepsilon\left(b_{2}\right)= \begin{cases}s \Lambda_{r}+\Lambda_{r+1} & \text { if } r>1, \\ s\left(\Lambda_{0}+\Lambda_{1}\right)+\Lambda_{2} & \text { if } r=1,\end{cases}
$$

which is of level $2 s+2$. 
Proof: The claim is easy to check explicitly.

Corollary 4.7 The KR crystal $B^{n, 2 s+1}$ of type $C_{n}^{(1)}$ is not perfect.

Proof: The $\{2, \ldots, n\}$-highest weight elements in the same component as $b_{1}$ and $b_{2}$ of Proposition 4.6 correspond to \pm -diagrams that are invariant under $\sigma$. Hence, by Definition 3.3, $b_{1}$ and $b_{2}$ are fixed points under $\sigma$. Combining this result with Proposition 4.5 proves that $B^{r, 2 s+1}$ is not perfect.

Proposition 4.8 There exists a bijection, induced by $\varepsilon$, from $B_{\min }^{r, 2 s}$ to $P_{s}^{+}$. Hence $B^{r, 2 s}$ is perfect of level $s$.

Proof: By Definition 3.3. $B^{r, s}$ of type $C_{n}^{(1)}$ is realized inside $B_{A_{2 n+1}^{(2)}}^{r, s}$ as the fixed points under $\sigma$. Under the embedding $S$, it is clear that a dominant weight $\Lambda=\ell_{0} \Lambda_{0}+\ell_{1} \Lambda_{1}+\cdots+\ell_{n+1} \Lambda_{n+1}$ of type $A_{2 n+1}^{(2)}$ is in the image if and only if $\ell_{0}=\ell_{1}$. Hence it is clear from the construction of the minimal elements for $A_{2 n+1}^{(2)}$ as described in Section 4.2 that the minimal elements corresponding to $\Lambda$ with $\ell_{0}=\ell_{1}$ are invariant under $\sigma$. By [22, Theorem 6.1] there is a bijection between all dominant weights $\Lambda$ of type $A_{2 n+1}^{(2)}$ with $\ell_{0}=\ell_{1}$ and $\operatorname{lev}(\Lambda)=2 s$ and minimal elements in $B_{A_{2 n+1}^{(2)}}^{r, 2 s}$ that are invariant under $\sigma$. Hence using $S$, there is a bijection between dominant weights in $P_{s}^{+}$of type $C_{n}^{(1)}$ and $B_{\min }^{r, 2 s}$.

The automorphism $\tau$ of the perfect $\mathrm{KR}$ crystal $B^{r, 2 s}$ is given by the identity.

\subsection{Type $C_{n}^{(1)}$ for $r=n$}

This case is treated in [14]. For the minimal elements, we follow the construction in Section 4.2 To every fundamental weight $\Lambda_{k}$ we associate a column tableau $T\left(\Lambda_{k}\right)$ of height $n$ whose entries are $k+1, k+$ $2, \ldots, n, \bar{n}, \ldots, \overline{n-k+1}(1,2, \ldots, n$ for $k=0)$ reading from bottom to top. Let $f\left(\Lambda_{k}\right)$ be defined such that $T\left(\Lambda_{k}\right)=f\left(\Lambda_{k}\right) b_{1}$, where $b_{k}$ is the highest weight tableau in $B\left(k \Lambda_{n}\right)$. Then the minimal element $b$ in $B^{n, s}$ such that $\varepsilon(b)=\Lambda=\sum_{i=0}^{n} \ell_{i} \Lambda_{i} \in P_{s}^{+}$is constructed as

$$
b=f\left(\Lambda_{n}\right)^{\ell_{n}} \cdots f\left(\Lambda_{1}\right)^{\ell_{1}} b_{s} .
$$

The automorphism $\tau$ is given by

$$
\tau\left(\sum_{i=0}^{n} \ell_{i} \Lambda_{i}\right)=\sum_{i=0}^{n} \ell_{i} \Lambda_{n-i}
$$

\subsection{Type $A_{2 n}^{(2)}$}

For type $A_{2 n}^{(2)}$ one may use the result of Naito and Sagaki [18, Theorem 2.4.1] which states that under their [18, Assumption 2.3.1] (which requires that $B^{r, s}$ for $A_{2 n}^{(1)}$ is perfect) all $B^{r, s}$ for $A_{2 n}^{(2)}$ are perfect. Here we provide a description of the minimal elements via the emebdding $S$ into $B_{C_{n}^{(1)}}^{r, 2 s}$.

Proposition 4.9 The minimal elements of $B^{r, s}$ of level s are precisely those that corresponding to doubled \pm -diagrams in $B_{C_{n}^{(1)}}^{r, 2 s}$. 
Proof: In Proposition 4.8 a description of the minimal elements of $B_{C_{n}^{(1)}}^{r, 2 s}$ is given. We have the realization of $B^{r, s}$ via the map $S$ from Definition 3.4. In the same way as in the proof of Proposition 4.4 one can show, that the minimal elements of $B_{C_{n}^{(1)}}^{r, 2 s}$ that correspond to doubled dominant weights are precisely those in the realization of $B^{r, s}$, hence $\varepsilon$ defines a bijection between $B_{\min }^{r, s}$ and $P_{s}^{+}$.

The automorphism $\tau$ is given by the identity.

4.8 Type $D_{n+1}^{(2)}$ for $r<n$

Proposition 4.10 Let $r<n$. There exists a bijection $B_{\min }^{r, s}$ to $P_{s}^{+}$, defined by $\varepsilon$. Hence $B^{r, s}$ is perfect.

Proof: This proof is analogous to the proof of Proposition 4.9 .

The automorphism $\tau$ is given by the identity.

\subsection{Type $D_{n+1}^{(2)}$ for $r=n$}

This case is already treated in [14], which we summarize below. As a $B_{n}$-crystal it is isomorphic to $B\left(s \Lambda_{n}\right)$. There is a description of its elements in terms of semistandard tableaux of $n \times s$ rectangular shape with letters from the alphabet $\mathcal{A}=\{1<2<\cdots<n<\bar{n}<\cdots<\overline{1}\}$. Moreover, each column does not contain both $k$ and $\bar{k}$. Let $c_{i}$ be the $i$ th column, then the action of $e_{i}, f_{i}(i=1, \ldots, n)$ is calculated through that of $c_{s} \otimes \cdots \otimes c_{1}$ of $B\left(\Lambda_{n}\right)^{\otimes s}$. With this realization the minimal element $b_{\Lambda}$ such that $\varepsilon\left(b_{\Lambda}\right)=\Lambda=\sum_{i=0}^{n} \ell_{i} \Lambda_{i} \in P_{s}^{+}$is given as follows. Let $x_{i j}(1 \leq i \leq n, j \in \mathcal{A})$ be the number of $j$ in the $i$ th row. Note that $x_{i j}=0$ unless $i \leq j \leq \overline{n-i+1}$. The table $\left(x_{i j}\right)$ of $b_{\Lambda}$ is then given by $x_{i i}=\ell_{0}+\cdots+\ell_{n-i}(1 \leq i \leq n), x_{i j}=\ell_{j-i}(i+1 \leq j \leq n), x_{i \bar{j}}=\ell_{j}+\cdots+\ell_{n}(n-i+1 \leq j \leq n)$. The automorphism $\tau$ is given by

$$
\tau\left(\sum_{i=0}^{n} \ell_{i} \Lambda_{i}\right)=\sum_{i=0}^{n} \ell_{i} \Lambda_{n-i}
$$

\section{References}

[1] G. Benkart, I. Frenkel, S-J. Kang, and H. Lee, Level 1 perfect crystals and path realizations of basic representations at $q=0$, Int. Math. Res. Not. 2006, Art. ID 10312, 28 pp.

[2] V. Chari, On the fermionic formula and the Kirillov-Reshetikhin conjecture, Internat. Math. Res. Notices 12 (2001) 629-654.

[3] V. Chari, A. Pressley, Quantum affine algebras and their representations, in Representations of groups, CMS Conf. Proc. 16, Amer. Math. Soc., Providence, RI (1995) 59-78.

[4] V. Chari, A. Pressley, Twisted quantum affine algebras, Comm. Math. Phys. 196 (1998) 461-476.

[5] G. Fourier, M. Okado, A. Schilling Kirillov-Reshetikhin crystals of non-exceptional type, Advances in Mathematics, to appear (arXiv:0810.5067 [math.RT])

[6] G. Fourier, M. Okado, A. Schilling Perfectness of Kirillov-Reshetikhin crystals of non-exceptional type, Contemp. Math., to appear (arXiv:0811.1604 [math.RT]) 
[7] G. Fourier, A. Schilling, M. Shimozono, Demazure structure inside Kirillov-Reshetikhin crystals, J. Algebra 309 (2007) 386-404.

[8] G. Hatayama, A. Kuniba, M. Okado, T. Takagi, Z. Tsuboi, Paths, crystals and fermionic formulae, MathPhys Odyssey 2001, 205-272, Prog. Math. Phys. 23, Birkhäuser Boston, Boston, MA, 2002.

[9] D. Hernandez, The Kirillov-Reshetikhin conjecture and solution of T-systems, J. Reine Angew. Math. 596 (2006) 63-87.

[10] D. Hernandez, Kirillov-Reshetikhin conjecture: The general case, preprint arXiv:0704.2838.

[11] J. Hong, S.-J. Kang, Introduction to quantum groups and crystal bases, Graduate Studies in Mathematics, 42, American Mathematical Society, Providence, RI, 2002. xviii+307 pp.

[12] V. G. Kac, “Infinite Dimensional Lie Algebras,” 3rd ed., Cambridge Univ. Press, Cambridge, UK, 1990.

[13] S-J. Kang, M. Kashiwara, K. C. Misra, T. Miwa, T. Nakashima, A. Nakayashiki, Affine crystals and vertex models, Int. J. Mod. Phys. A 7 (suppl. 1A) (1992), 449-484.

[14] S.-J. Kang, M. Kashiwara, K. C. Misra, T. Miwa, T. Nakashima, A. Nakayashiki, Perfect crystals of quantum affine Lie algebras, Duke Math. J. 68 (1992) 499-607.

[15] M. Kashiwara, On crystal bases of the q-analogue of universal enveloping algebras, Duke Math. J. 63 (1991), 465-516.

[16] M. Kashiwara, K. C. Misra, M. Okado, D. Yamada, Perfect crystals for $U_{q}\left(D_{4}^{(3)}\right)$, J. Alg. 317 (2007) 392-423.

[17] M. Kashiwara, T. Nakashima, Crystal graphs for representations of the q-analogue of classical Lie algebras, J. Alg. 165 (1994) 295-345.

[18] S. Naito, D. Sagaki, Construction of perfect crystals conjecturally corresponding to KirillovReshetikhin modules over twisted quantum affine algebras, Comm. Math. Phys. 263 (2006), no. 3, 749-787.

[19] H. Nakajima, t-analogues of q-characters of Kirillov-Reshetikhin modules of quantum affine algebras, Represent. Theory 7 (2003) 259-274.

[20] M. Okado, Existence of crystal bases for Kirillov-Reshetikhin modules of type D, Publ. RIMS 43 (2007) 977-1004.

[21] M. Okado, A. Schilling, Existence of Kirillov-Reshetikhin crystals for nonexceptional types, Representation Theory 12 (2008) 186-207.

[22] A. Schilling, Combinatorial structure of Kirillov-Reshetikhin crystals of type $D_{n}^{(1)}, B_{n}^{(1)}, A_{2 n-1}^{(2)}$ J. Algebra 319 (2008) 2938-2962.

[23] M. Shimozono, Affine type A crystal structure on tensor products of rectangles, Demazure characters, and nilpotent varieties, J. Algebraic Combin. 15 (2002), no. 2, 151-187. 
[24] S. Yamane, Perfect crystals of $U_{q}\left(G_{2}^{(1)}\right)$, J. Algebra 210 (1998), no. 2, 440-486. 